\title{
Robust German Noun Chunking With a Probabilistic Context-Free Grammar
}

\author{
Helmut Schmid and Sabine Schulte im Walde* \\ Institut für Maschinelle Sprachverarbeitung \\ Universität Stuttgart \\ Azenbergstraße 12, 70174 Stuttgart, Germany \\ \{schmid, schulte\}@ims.uni-stuttgart.de
}

\begin{abstract}
We present a noun chunker for German which is based on a head-lexicalised probabilistic contextfree grammar. A manually developed grammar was semi-automatically extended with robustness rules in order to allow parsing of unrestricted text. The model parameters were learned from unlabelled training data by a probabilistic context-frce parser. For extracting noun chunks, the parser generates all possible noun chunk analyses, scores them with a novel algorithm which maximizes the best chunk sequence criterion, and chooses the most probable chunk sequence. An evaluation of the chunker on 2,140 hand-annotated noun chunks yielded $92 \%$ recall and $93 \%$ precision.
\end{abstract}

\section{Introduction}

A noun chunker marks the noun chunks in a sentence as in the following cxample:

(Wirtschaftsbosse) mit (zweifelhaftem Ruf) cconomy chefs with doubtable reputation sind an (der in (Engpässen) angewandten Führung) are in the in bottlenecks applied guidance (des Landes) beteiligt.

of the country involved.

'Leading economists with doubtable reputations are involved in guiding the country in times of bottlenecks.'

A tool which identifies noun chunks is useful for term cxtraction (most technical terms are nouns or complex noun groups), for lexicographic purposes (see (Tapanainen and Järvinen, 1998) on syntactically organised concordancing), and as index terms for information retrieval. Chunkers may also mark other types of chunks like verb groups, adverbial phrases or adjectival phrases.

Several methods have been developed for noun chunking. Church's noun phrase tagger (Church, 1988), one of the first noun chunkers, was based on a Hidden Markov Model (HMM) similar to those used

\footnotetext{
* Thanks to Mats Rooth and Uli Heid for many helpful comments.
}

for part-of-spech tagging. Another HMM-based approach has been developed by Mats Rooth (Rooth, 1992). It integrates two HMMs; one of them models noun chunks internally, the other models the context of noun chunks. Abney's cascaded finitcstate parser (Abney, 1996) also contains a processing step which recognises noun chunks and other types of chunks. Ramshaw and Marcus (Ramshaw and Marcus, 1995) successfully applied Eric Brill's transformation-based learning method to the chunking problem. Voutilainen's NPtool (Voutilainen, 1993) is based on his constraint-grammar system. Finally, Brants (Brants, 1999) described a German chunker which was implemented with cascaded Markov Models.

In this paper, a probabilistic context-free parser is applied to the nom chunking task. The German grammar used in the experiments was semiautomatically extended with robustmess rules in order to be able to process arbitrary input. The grammar parameters were trained on unlabelled data. A novel algorithm is used for noun chunk extraction. It maximises the probability of the chunk set.

The following section introduces the grammar framework, followed by a description of the chunking algorithm in section 3 , and the experiments and their evaluation in section 4 .

\section{The Grammar}

The German grammar is a head-lexicalised probabilistic context-free grammar. Section 2.1 defines probabilistic context-free grammars and their headlexicalised refinement. Section 2.2 introduces our grammar architecture, focusing on noun chunks. The robustness rules for the chunker are described in section 2.3.

\section{1 (Head-Lexicalised) Probabilistic Context-Free Grammars}

A probabilistic context-free grammar ( $\mathrm{PCFG}$ ) is a context-free grammar which assigns a probability $P$ to each context-free grammar rule in the rule set R. The probability of a parse tree $T$ is defined as $\prod_{r \in R} P(r)^{|r|}$, where $|r|$ is the number of times rule $r$ was applied to build $T$. The parameters of 
PCFGs can be learned from unparsed corpora using the Inside-Outside algorithm (Lari and Young, $1990)$.

Head-lexicalised probabilistic context-free grammars (H-L PCFG) (Carroll and Rooth, 1998) oxtend the PCFG approach by incorporating information about the lexical head of constituents into the probabilistic model. ${ }^{1}$ Each node in a parse of a $\mathrm{H}$ $\mathrm{L}$ ?'CFG is labelled with a category and the lexical head of the category. A H-L PCFG rule looks like a $\mathrm{PCFG}$ rule in which one of the daughters has been marked as the head. The rule probabilities $P_{\text {rule }}(C \rightarrow \alpha \mid C)$ aro replaced by lexicalised rulo probabilities $P_{\text {rule }}(C \rightarrow \alpha \mid C, h)$ where $h$ is the lexical head of the mother constituent $C$. The probability of a rule therefore depends not only on the category of the mother node, but also on its lexical head. Assume that the grammar has two rules VP $\rightarrow$ V NP and VP $\rightarrow$ V. Then the transitive verb buy should have a higher probability for the former rule whoreas the latier rule should be moro likely for intransitive verbs like slecp. IJ-I, PCFGs incorporate another type of parameters called lexical choice probabilitics. The lexical choice probability $P_{\text {choice }}\left(h_{d} \mid C_{d}, C_{m}, h_{m}\right)$ represents the probability that a node of category $C_{d}$ with a mother node of category $C_{m}$ and lexical head $h_{m}$ bears the lexical head $h_{d}$. The probability of a parse tree is obtained by multiplying lexicalised rule probabilities and lexical choice probabilities for all nodes. Since it is possible to transform H-L P'CFGs into PCFG's, the PCTG algorithms are applicable to H-T J'Cl'Gs.

\subsection{Noun Chunks in the German Grammar}

Currently, the Geman grammar contains 4,619 rules and covers $92 \%$ of our 15 million words of verb final and relative clauses ${ }^{2}$. The structural noun chunk concept in the grammar: is defined according; to Abney's chunk style (Abney, 1991) who describes chunks as syntactic units which correspond in some way to prosodic patterns, containing a content word surrounded by some function word(s): all words from the beginning of the noun phrase to the head nown are included. ${ }^{3}$ The different kinds of nom chunks covered by our grammar are listed below and illustrated with examples:

- a combination of a non-obligatory determiner, optional adjectives or cardinals and the noun

\footnotetext{
${ }^{1}$ Other types of lexicalised PClFGs have been described in (Charniak, 1997), (Collins, 1997), (Goodman, 1997), (Chelba and Jelinek, 1998) and (Fisner and Satta, 1999).

2'Ihe restricted corpora were extracted automatically from the IIuge German Corpus ( $H G(Y)$, a collection of German newspapers as well as specialised magazines for industry, law, computer science.

${ }^{3}$ As you will see below, there is one exception, noun chunks refined by a proper name, which end with the name instead of the liead noun.
}

itssolf:

(1.) cine gute Idee a good idea

(2) vielen Menschen for many people

(3) deren künstliche Stimme whose artificial voice

(4) elf Ladungen eleven cargos

(5) Wasser water

and prepositional phrases where the definite article of the cmbedded noun chunk is morphologically combined with a preposition, so the purc noun chunk could not be separated:

(6) zum Schluss at the end

- personal pronouns: ich (I), mir (me)

- reflexivo pronouns: mich (myself), sich (himsolf/horself/itsclf)

- possessive pronouns:

(7) Meins ist sauber. Minc is cloan.

- demonstrative pronouns:

(8) Jener fährt vicl schnollor. That one goes much faster.

- indefinite pronoms:

(9) Einige sind durchgefallen. Some failed.

- relative pronouns:

(10) Ich mag Menschen, die ehrlich sind. I like people who are honest.

- nominalised adjectives: Wichtigem (important things)

- proper names: Christoph Kolumbus

- a noun clinnk refined by a proper name:

(1.) der Eroberer Christoph Kolumbus the conquerer Christoph Columbus

- cardinals indicating a ycar:

(12) Ich begann 1996.

I started 1996.

The chunks may be recursive in case they appear as complement of an adjectival phrase, as in (der (im Regen) wartende Sohn) (the son who was waiting in the rain).

Noun chunks have features for casc, without further agreement features for nouns and verbs. The case is constrained by the function of the noun chunk, as verbal or adjectival complement with nominative, accusative, dative or genitive case, as modifier with genitive case, or as part of a prepositional 
phrase (also in the special case representing a prepositional phrase itself) with accusative or dative case.

Both structure and case of noun phrases may be ambiguous and have to be disambiguated:

- ambiguity concerning structure: diesen (this) is disregarding the context a demonstrative pronoun ambiguous between representing a standalone noun chunk (cf. example (8)) or a determiner within a noun chunk (cf. example (2))

- ambiguity concerning case:

die Beiträge (the contributions) is disregarding the context ambiguous between nominative and accusative case

The disambiguation is learned during grammar training, since the lexicalised rule probabilities as well as the lexical choice probabilities tend to enforce the correct structure and case information. Considering the above examples, the trained grammar should be able to parse diesen Krieg (this war) as one noun chunk instead of two (with diesen representing a standalone noun chunk) because of (i) the preferred use of demonstrative pronoms as determiners ( $\rightarrow$ lexicalised rule probabilities), and (ii) the lexical coherence between the two words ( $\rightarrow$ lexical choice probabilities); in a sentence like er zahlte die Beiträge (he paid the contributions) the accusative case of the latter noun chunk should be identified because of the lexical colnerence between the verb zahlen (pay) and the lexical head of the subcategorised noun phrase Beitrag (contribution) as related direct object head $(\rightarrow$ lexical choice probabilitics)

\subsection{Robustness Rules}

The German grammar covers over $90 \%$ of the clauses of our verb final and relative clause corpora. This is sufficient for the extraction of lexical information, e.g. the subcategorisation of verbs (see (Beil et al., 1999)). For chunking; however, it is usually necessary to analyse all sentences. Therefore, the grammar was augmented with a set of robustness rules. Three types of robustness rules have been considered, namely unigram rules, bigram rules and trigram rules.

Unigram rules are rules of the form $X \rightarrow Y P X$, where YP is a grammatical category and $X$ is a new category. If such a rule is added for each grammar category $^{4}$, the coverage is $100 \%$ because the grammar is then able to generate any sequence of category labels. In practice, some of the rules can be omitted while still retaining full coverage: $\mathrm{c.g}$. the rule $\mathrm{X} \rightarrow$

\footnotetext{
${ }^{4}$ Also needed are two rules which start and terminate the "X chain". We used the rules TOP $\rightarrow$ START $X$ and $X \rightarrow$ END. START and END expand to SGML tags which mark the beginning and the end of a sentence, respectively.
}

ADV $X$ is not necessary if the grammar already contains the rules ADVP $\rightarrow$ ADV and $X \rightarrow$ ADVP $X$. Unigram rules are insensitive to their context so that all permutations of the categories which are gencrated by the $\mathrm{X}$ chain have the same probability.

The second type of robustness rules, called trigram rules (Carroll and Rooth, 1998) is more context sensitive. Trigram rules have the form $X: Y \rightarrow Y Y: Z$ where $X, Y, Z$ are categories and $X: Y$ and $Y: Z$ are new categories. Trigram rules choose the next category on the basis of the two preceding categories. Therefore the number of rules grows as the number of categories raises to the third power. For example, 125,000 trigram rules are needed to gencrate 50 different categories in arbitrary order.

Since unigram rules are context insensitive and trigram rules are too numerous, a third type of robustness rules, called bigram rules, was developed. A bigram rule actually consists of two rules, a rule of the form $: Y \rightarrow Y Y$ : which gencrates the constituent $Y$ deterministically, and a rule $Y: \rightarrow: Z$ which selects the next constituent $Z$ based on the current one. Given $n$ categories, wo obtain $n$ rules of the first form and $n^{2}$ rules of the second form. Even when categories which directly project to some other category were omitted in the generation of the bigram rules for our German grammar, the number of rules was still fairly large. Hence we generalised some of the grammatical catcgories by adding additional chain rules. For example, the prepositional phrase categories PP.Akk:an, PP.Akk:auf, PP.Akk:gegen etc. were generalised to PPX by adding the rules PPX $\rightarrow$ PP. Akk: an etc. Instcad of $n+1$ bigram rules for cach of the 23 prepositional categories, we now obtained only $n+2$ rules with the new category PPX. Altogether, 3,332 robustness rules wore added.

\section{Chunking}

A head-lexicalised probabilistic context-free parser, called LoPar (Schmid, 1999), was used for parsing. The functionality of LoPar encompasses purely symbolic parsing as woll as Viterbi parsing, insideoutside computation, POS tagging, chunking and training with PCFGs as well as H-L PCFGs. Bccause of the large number of parameters in particular of H-L PCFGs, the parser smoothes the probability distributions in order to avoid zero probabilities. The absolute discounting method (Ney et al., 1994) was adapted to fractional counts for this purpose. LoPar also supports lemmatisation of the lexical heads of a H-L PCFG. The input to the parser consists of ambiguously tagged words. The tags are provided by a German morphological analyser (Schiller and Stöckert, 1995).

The best chunk set of a sentence is defined as the set of chunks (with category, start and end position) 
for which the sum of the probabilities of all parses which contain exactly that clnnk set is maximal. The chunk set of the most likely parse (i.e. Viterbi parse) is not necessarily the best chunk set according to this definition, as the following P CFG shows.

$\begin{array}{llll}\mathrm{S} \rightarrow \mathrm{A} & 0.6 & \mathrm{~B} \rightarrow \mathrm{x} & 1.0 \\ \mathrm{~S} \rightarrow \mathrm{B} & 0.4 & \mathrm{C} \rightarrow \mathrm{x} & 1.0 \\ \mathrm{~A} \rightarrow \mathrm{C} & 0.5 & \mathrm{D} \rightarrow \mathrm{x} & 1.0 \\ \mathrm{~A} \rightarrow \mathrm{D} & 0.5 & & \end{array}$

This grammar generaties the three parse trees (S $(A(C x))),(S(A(D x)))$, and $(S(B x))$. The parse tree probabilitics are $0.3,0.3$ and 0.4 , respectively. The last parse is therefore the Viterbi parse of $x$. Now assume that $\{A, B\}$ is the set of chunk categories. The most likely chunk set is then $\{(A, 0,1)\}$ because the stum of the probabilitios of all parses which contain $\mathrm{A}$ is 0.6 , whereas the sum over the probabilitios of all parses containing $B$ is only 0.4 .

computeChunks is a slightly simplifice pseudocole version of the actual chunking algorithm:

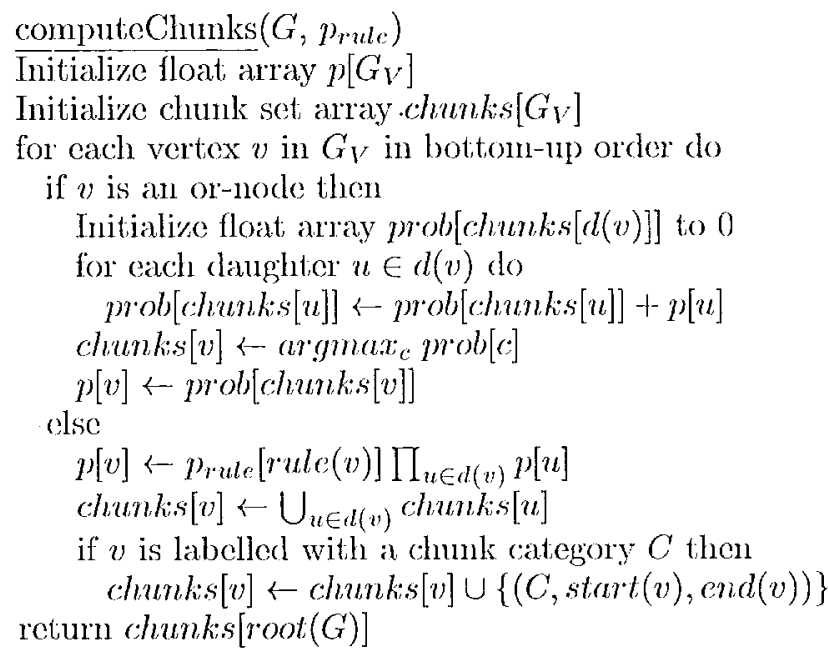

computechunks takes two arguments. The first algument is a parse forest $G$ which is represented as an and-or-graph. $G_{V}$ is the set of vertices. The second argument is the rule probability vector. $d$ is a function which returns the daughters of a vertex. The algorithm computes the best chunk set chunks [v] and the corresponding probability $p[v]$ for all vertices $v$ in bottom-up order. chunks[d(v)] returns the set of chunk sets of the daughter nodes of vertex $v$. rule $(v)$ returns the rule which created $v$ and is only defined for and-nodes. start $(v)$ and end $(v)$ roturn the start and end position of the constituent represented by $v$.

The chunking algorithm was experimentally comparcl with chunk extraction from Viterbi parses. In 35 out of 41 evaluation runs with different parameter settings ${ }^{5}$, the f-score of the chunking algorithm

\footnotetext{
5'The runs differed wrt. training strategy and number of iterations. See section 4 for details.
}

was better than that of the Viterbi algorithm. The average f-score of the chunking algorithm was $84.7 \%$ compared to $84.0 \%$ for the Viterbi algorithm.

\section{Experiments}

We performed two main chunking experiments. Initially, the parser trained the chunk grammar based on the restricted grammar described in section 2 according to four different training strategies. A preferred training strategy was then applied to investigate the potential of grammar refinement and $\mathrm{cx}$ tonded training data.

\subsection{Training}

In the first experiment, the chunker version of the grammar was trained on a corpus comprising a 1 million word subcorpus of rolative clauses, a 1 million word subcorpus of verb final clauses and 2 million words of consecutive text. All data had been extracted from the Huge German Corpus. The test lata used for the later evaluation was not included in the training corpus.

For training strategy 1, the chunker grammar was first trained on the whole corpus in unlexicalised mode, i.e. like a PCFG. The parameters were reestimated once in the middle and once at the end of the corpus. In the next step, the grammar was lexicalised, i.c. the parser computed the parse probabilities with the unlexicalised model, but extracted froquencies for the lexicalised model. These frequencies were summed over the whole corpus. Three more iterations on the whole corpus followed in which the parameters of the lexicalised model were reestimated.

The parancters of the unlexicalised chnnker grammar were initialised in the following way: a frequency of 7500 was assigned to all original grammar rules and 0 to the majority of robustness rules. The parameters were then estimated on the basis of these frequencies. Bocause of the smoothing, the probabilities of the robustmess rules were small but not zoro.

For training strategy 2, the chunker rules were initialised with frequencies from a grammar without robustness rule cxtensions, which had been trained unlexicalised on a 4 million subcorpus of verb final clauses and a 4 million word subcorpus of relative clauses.

Training strategy 3 again set the frequency of the original rules to 7500 and of the robustness rules to 0 . The parser trained with three unlexicalised iterations over the whole training corpus, reestimating the parameters only at the end of the corpus, in order to find out whether the lexicalised probabilistic parser had been better than the fully trained unlexicalised parser on the task of chunk parsing. Training strategy 4 repeated this procedure, but with initial- 
ising the chunker frequencies on basis of a trained grammar.

For cach training strategy, further iterations were added until the precision and recall values ceased to improve.

For the second part of the experiments, the base grammar was extended with a fow simple verb-first and verb-second clause rules. Strategy 4 was applied for training the chunker

(A) on the same training corpus as before, i.c. 2 million words of relative and verb final clauses, and 2 million words of umestricted corpus data from the HGC,

(B) on a training corpus consisting of 10 million words of unrestricted corpus data from the HGC.

\subsection{Evaluation}

The evaluation of the chunker was carried out on noun chunks from 378 unrestricted sentences from the German newspaper Frankfurter Allgemeine Zeitung (FAZ). Two persons independently annotated all noun chunks in the corpus - a total of 2,140 noun chunks-, according to the noun chunk definition in section 2.2 , without considering grammar coverage, i.e. noun chunks not actually covered by the grammar (e.g. noun chunk ellipsis such as die kleinen []$_{N}$ ) were annotated as such. As labels, wo used the identifier NC plus case information: NC. Nom, NC.Acc, NC.Dat, NC.Gen. In addition, we included identifiers for prepositional phrases where the preposition is morphologically merged with the definite article, (cf. cxample (6)), also including case information: PNC.Acc, PNC.Dat.

For each training strategy described in section 4.1 we evaluated the chunker before the training process and after cach training iteration: the model in its current training state parsed the test sentences and extracted the most probable chunk sequence as defined in section 3 . We then compared the extracted noun chunks with the hand-annotated data, according to

- the range of the chunks, i.e. did the chunker find a chunk at all?

- the range and the identifier of the chunks, i.e. did the chunker find a chunk and identify the correct syntactic category and case?

Figures 1 and 2 display the results of the evaluation in the first experiment, ${ }^{6}$ according to noun chunk range only and according to noun chunk range, syntactic category and case, respectively. Bold font highlights the best versions.

Training strategy 2 with two iterations of lexicalised training produced the best f-scores for noun

\footnotetext{
${ }^{6}$ The lexicalised chunker versions obtained by strategy 2 were also utilised for parsing the test sentences unlexicalised.
}

chunk boundary recognition if unlexicalised parsing was done. The respective precision and recall values were $93.06 \%$ and $92.19 \%$. For recognising noun chunks with range, category and case, the best chunker version was created by training strategy 4 , after five iterations of unlexicalised training; precision and recall values were $79.28 \%$ and $76.75 \%$, respectively.

From the experimental results, we can conclude that:

1. initialisation of the chunker grammar frequoncies on the basis of a trained grammar improves the untrained version of the chunker, but the difference vanishes in the training process

2. unlexicalised parsing is sufficient for noun chunk extraction; for extraction of chunks with case information, unlexicalised training turned out to be even more successful than a combination with lexicalised training

Figures 3 and 4 display the results of the evaluation concerning the second experiment, compared to the initial values from the first experiment.

Extending the base grammar and the training corpus slightly increased precision and recall values for recognising noun chunks according to range only. The main improvement was in noun chunk recognition according to range, category and case: precision and recall values increased to $83.88 \%$ and $83.21 \%$, respectively.

\subsection{Failure Analysis}

A comparison of the parsed noun chunks with the annotated data showed that failure in detecting a noun chunk was mainly caused by proper names, for example Netanjahu, abbreviations like OSZE, or composita like South China Morning Post. The diversity of proper names makes it difficult for the chunker to learn thom properly. On the one hand, the lexical information for proper names is unreliable because many proper names were not recognised as such. On the other hand, most proper names arc too rare to learn reliable statistics for them.

Minor mistakes were caused by (a) articles which are morphologically identical to nom chunks consisting of a pronoun, for example den Rentnern (the pensioners dat) was analysed as two noun chunks, den (demonstrative pronoun) and Rentnern, (b) capital letter confusion: since German nouns typically start with capital lotters, sentence beginnings are wrongly interpreted as nouns, for example Würden as the conditional of the auxiliary werden (to become) is interpreted as the dative case of Würde (dignity), (c) noun chunk internal punctuation as in seine 'Partner' (his ' partners').

Failure in assigning the correct syntactic category and case to a noun chunk was mainly caused by (a) assigning accusative case instcad of nominative casc, and (b) assigning dative case or nomina- 


\begin{tabular}{|c|c|c|c|c|c|c|c|c|c|c|}
\hline & \multicolumn{2}{|c|}{ Strategy 1} & \multicolumn{2}{|c|}{ Strategy 2} & \multicolumn{2}{|c|}{-parsed unlex- } & \multicolumn{2}{|c|}{ Strategy 3} & \multicolumn{2}{|c|}{ Strategy 4} \\
\hline & prec & rec & prec & rec & proc & rec & prec & $\mathrm{rec}$ & prec & rec \\
\hline untratined & $83.63 \%$ & $83.63 \%$ & $90.22 \%$ & $90.18 \%$ & $90.22 \%$ & $90.18 \%$ & $83.63 \%$ & $83.63 \%$ & $90.22 \%$ & $90.18 \%$ \\
\hline unlex 1 & $91.33 \%$ & $89.62 \%$ & $92.84 \%$ & $01.58 \%$ & $92.84 \%$ & $91.58 \%$ & $91.33 \%$ & $89.62 \%$ & $92.84 \%$ & $91.58 \%$ \\
\hline unlex2 & & & & & & & $92.55 \%$ & $90.04 \%$ & $93.01 \%$ & $91.49 \%$ \\
\hline unlex3 & & & & & & & $92.78 \%$ & $90.22 \%$ & $92.95 \%$ & $91.25 \%$ \\
\hline unlex 4 & & & & & & & & & $93.09 \%$ & $90.79 \%$ \\
\hline unlex 5 & & & & & & & & & $93.29 \%$ & $90.32 \%$ \\
\hline $\operatorname{lex} 0$ & $89.13 \%$ & $89.71 \%$ & $90.12 \%$ & $90.41 \%$ & $93.01 \%$ & $91.49 \%$ & & & & \\
\hline lex1. & $88.37 \%$ & $89.52 \%$ & $88.97 \%$ & $89.76 \%$ & $93.02 \%$ & $91.67 \%$ & & & & \\
\hline $\operatorname{lex} 2$ & $88.25 \%$ & $89.57 \%$ & $89.79 \%$ & $90.46 \%$ & $93.06 \%$ & $92.19 \%$ & & & & \\
\hline $\operatorname{lox} 3$ & $88.17 \%$ & $89.62 \%$ & $89.42 \%$ & $90.13 \%$ & $93.05 \%$ & $92.05 \%$ & & & & \\
\hline
\end{tabular}

Figure 1: Comparing training strategies: nom chunk evaluation according to rango only

\begin{tabular}{|c|c|c|c|c|c|c|c|c|c|c|}
\hline & \multicolumn{2}{|c|}{ Strategy 1} & \multicolumn{2}{|c|}{ Strategy 2} & \multicolumn{2}{|c|}{ parsed unlex- } & \multicolumn{2}{|c|}{ Strategy 3} & \multicolumn{2}{|c|}{ Strategy 4} \\
\hline & prec & res & procs & rec & prec & rec & prec & $\mathrm{roc}$ & prec & $\mathrm{rec}$ \\
\hline untrained & $63.52 \%$ & $63.52 \%$ & $72.02 \%$ & $71.98 \%$ & $72.02 \%$ & $71.98 \%$ & $63.52 \%$ & $63.52 \%$ & $72.02 \%$ & $71.98 \%$ \\
\hline minlex 1 & $74.50 \%$ & $73.11 \%$ & $75.87 \%$ & $74.81 \%$ & $75.87 \%$ & $71.84 \%$ & $74.50 \%$ & $73.11 \%$ & $75.87 \%$ & $74.84 \%$ \\
\hline unlex2 & & & & & & & $76.88 \%$ & $74.79 \%$ & $78.27 \%$ & $76.99 \%$ \\
\hline manlex3 & & & & & & & $77.97 \%$ & $75.82 \%$ & $78.70 \%$ & $77.27 \%$ \\
\hline unlex4 & & & & & & & & & $78.80 \%$ & $76.85 \%$ \\
\hline unlex5 & & & & & & & & & $79.28 \%$ & $76.75 \%$ \\
\hline $\operatorname{lex} 0$ & $73.68 \%$ & $73.15 \%$ & $75.10 \%$ & $75.35 \%$ & $78.27 \%$ & $76.99 \%$ & & & & \\
\hline $\operatorname{lex} 1$ & $72.02 \%$ & $72.97 \%$ & $74.69 \%$ & $75.35 \%$ & $77.27 \%$ & $76.15 \%$ & & & & \\
\hline $\operatorname{lex} 2$ & $72.76 \%$ & $73.85 \%$ & $75.26 \%$ & $75.82 \%$ & $77.48 \%$ & $76.75 \%$ & & & & \\
\hline lex:3 & $71.97 \%$ & $73.15 \%$ & $75.03 \%$ & $75.63 \%$ & $77.45 \%$ & $70.61 \%$ & & & & \\
\hline
\end{tabular}

Figue 2: Comparing training strategies: noun chunk evaluation according to range and label

tive case instead of accusative casc. The confusion between nominative and accusative case is due to the fact that, both cases are expressed by identical morphology in the fominine and nentral genders in German. The morphologic: similarity between accubative and dative is less substantial, but especially proper names and bare nouns are still subject to confusion. As the evaluation results show, the distinction between the cases could be learned in general, but morphological similarity and in addition the rolatively free word order in German impose high demands on the necessary probability model.

\section{Summary}

Wo presented a German noun chunker for nuncstricted text. The chunker is based on a headlexicalised probabilistic context-frec grammar and trained on unlabelled datia. The base grammar was semi-automatically augmented with robustncss rules in order to cover unrestricted input. An algorithm for chunk extraction was developed which maximises the probability of the chunk sets rather than the probability of single parses like the Viterlbi algorithm.

German noun chunks wore detected with $93 \%$ precision and $92 \%$ recall. Asking tho chunker to additionally identify the syntactic category and the case of the chunks resulted in recall of $83 \%$ and precision of $84 \%$. A comparison of different training strate- gies showed that mlexicalised parsing information was sufficient for noun chunk extraction with and without case information. 'The baso grammar played an important role in the chunker development: (i) building the chunker on the basis of an alseady trained grammar improved the clunker rules, and (ii) refining the base grammar with even simple verbfirst and verb-second rules improved accuracy, so it should be worthwhile to further extend the grammar rules. Increasing the amount of training data also improved noun chunk recognition, especially case disambiguation. Better heuristics for guessing the parts-of-specch of unknown words should further improve the noun chunk recognition, since many orrors were caused by 1unknown words.

\section{References}

Steven Abney. 1991. Parsing by Chunks. In Robert; Berwick, Steven Abney, and Carol Tenny, editors, Principle-Based Parsing. Kluwer Academic Publishers.

Stevon Abney. 1996. Partial Parsing via FiniteState Cascades. In Procedings of the ESSLLI'96 Robust Parsing Worlishop.

Tranz Beil, Glem Carroll, Detlef Prescher, Stefan Riczler, and Mats Rooth. 1999. Inside-Outside Estimation of a Lexicalized PCFG for German. In Proceedings of the 37th Annual Mecting of the $A C L$, pages 269-276. 


\begin{tabular}{|c|c|c|c|c|c|c|}
\hline & \multicolumn{6}{|c|}{ Strategy 4} \\
\hline & \multicolumn{2}{|c|}{ Initial } & \multicolumn{2}{|c|}{$\mathrm{A}$} & \multicolumn{2}{|c|}{ B } \\
\hline & prec & rec & prec & rec & prec & rec \\
\hline untrained & $90.22 \%$ & $90.18 \%$ & $90.43 \%$ & $90.60 \%$ & $90.43 \%$ & $90.60 \%$ \\
\hline unlex 1 & $92.84 \%$ & $91.58 \%$ & $91.65 \%$ & $91.35 \%$ & $91.52 \%$ & $91.35 \%$ \\
\hline unlex2 & $93.01 \%$ & $91.49 \%$ & $92.45 \%$ & $91.58 \%$ & $91.89 \%$ & $91.67 \%$ \\
\hline tunlex3 & $92.95 \%$ & $91.25 \%$ & $92.64 \%$ & $91.21 \%$ & $92.21 \%$ & $91.86 \%$ \\
\hline tunlex4 & $93.09 \%$ & $90.79 \%$ & $93.11 \%$ & $91.07 \%$ & $92.73 \%$ & $91.86 \%$ \\
\hline unlex 5 & $93.29 \%$ & $90.32 \%$ & $93.20 \%$ & $91.02 \%$ & $92.73 \%$ & $91.86 \%$ \\
\hline unlex6 & & & & & $92.91 \%$ & $91.96 \%$ \\
\hline unlex 7 & & & & & $92.83 \%$ & $92.10 \%$ \\
\hline
\end{tabular}

Figure 3: Grammar and training data extensions: noun chunk evaluation according to range only

\begin{tabular}{|l||r|r||r|r||r|r|}
\hline \multicolumn{1}{|c|}{} & \multicolumn{3}{c|}{ Stratcgy 4 } & \multicolumn{2}{c|}{ A } & \multicolumn{2}{c|}{ B } \\
\cline { 2 - 6 } & prec & rec & prec & rec & prec & \multicolumn{1}{c|}{ rec } \\
\hline untrained & $72.02 \%$ & $71.98 \%$ & $74.42 \%$ & $74.56 \%$ & $74.42 \%$ & $74.56 \%$ \\
unlex1 & $75.87 \%$ & $74.84 \%$ & $78.51 \%$ & $78.25 \%$ & $77.60 \%$ & $77.46 \%$ \\
unlex2 & $78.27 \%$ & $76.99 \%$ & $80.74 \%$ & $\mathbf{7 9 . 9 8 \%}$ & $79.89 \%$ & $79.70 \%$ \\
unlex3 & $78.70 \%$ & $\mathbf{7 7 . 2 7 \%}$ & $81.24 \%$ & $\mathbf{7 9 . 9 8 \%}$ & $81.17 \%$ & $80.87 \%$ \\
unlex4 & $78.80 \%$ & $76.85 \%$ & $81.83 \%$ & $80.03 \%$ & $82.41 \%$ & $81.67 \%$ \\
unlex5 & $\mathbf{7 9 . 2 8 \%}$ & $76.75 \%$ & $\mathbf{8 1 . 8 5 \%}$ & $79.93 \%$ & $82.53 \%$ & $81.76 \%$ \\
unlex6 & & & & & $82.94 \%$ & $82.09 \%$ \\
unlex7 & & & & & $\mathbf{8 3 . 8 8 \%}$ & $\mathbf{8 3 . 2 1 \%}$ \\
\hline
\end{tabular}

Figure 4: Grammar and training data extensions: noun chunk evaluation according to range and label

Thorsten Brants. 1999. Cascaded markov models. In Proceedings of EACI' 99 .

Glenn Carroll and Mats Rooth. 1998. Valence Induction with a Head-Lexicalized PCFG. In Proccedings of Third Conference on Empirical Mcthods in Natural Language Processing.

Eugene Charniak. 1997. Statistical Parsing with a Context-Free Grammar and Word Statistics. In Proceedings of the 14th National Conference on Artificial Intelligence.

Ciprian Chelba and Frederick Jelinek. 1998. Exploiting Syntactic Structure for Language Modeling. In Proceedings of the 36th Annual Meeting of the $A C L$.

Kenneth W. Church. 1988. A Stochastic Parts Program and Noun Phrase Parser for unrestricted Text. In Proceedings of the Second Conference on Applied Natural Language Processing, pages 136143.

Michael Collins. 1997. Three Gencrative, Lexicalised Models for Statistical Parsing. In Proceedings of the 35th Annual Meeting of the ACL.

Jason Eisner and Giorgio Satta. 1999. Efficient Parsing for Bilexical Context-Free Grammars and Head Automaton Grammars. In Proceedings of the 37th Annual Meeting of the ACL, pages 457464.

Joshua Goodman. 1996. Parsing Algorithms and Metrics. In Proceedings of the 34th Annual Meeting of the $A C L$, pages 177-183.

Joshua Goodman. 1997. Probabilistic Feature
Grammars. In Proceedings of the 5th International Workshop on Parsing Technologies, pages 89-100.

K. Lari and S. Young. 1990. The Estimation of Stochastic Context-Free Grammars using the Inside-Ontside Algorithm. Computation Speech. and Language Processing, 4:35-56.

Hermann Ney, U. Essen, and R. Kneser. 1994. On Structuring Probabilistic Dependencies in Stochastic Language Modelling. Computer Speech and Language, 8:1-38.

L. Ramshaw and M. Marcus. 1995. Text Chunking using Transformation-Based Lcarning. In Proceedings of the Third Workshop on Very Large Corpora, pages 82-94.

Mats Rooth. 1992. Statistical NP Tagging. Unpublished manuscript.

Anne Schiller and Chris Stöckert, 1995. DMOR. Institut für Maschinelle Sprachverarbeitung, Universität Stuttgart.

Helmut Schmid. 1999. Lopar: Design and Implomentation. Technical report, Institut für Maschinelle Sprachverarbeitıng, Universität Stuttgart.

Pasi Tapanainen and Timo Järvinen. 1998. Dependency Concordances. International Journal of Lexicography, 11(3):187-203.

Atro Voutilainen. 1993. NPtool, a Detector of English Noun Phrases. In Proceedings of the Workshop on Very Large Corpora, pages 48-57. 